\title{
Tangence
}

\section{L'envers fatal de la curiosité}

\section{Lecture sémiotique d'un fait divers passionnel}

\section{Louise Milot}

Numéro 37, septembre 1992

Autopsie du fait divers

URI : https://id.erudit.org/iderudit/025726ar

DOI : https://doi.org/10.7202/025726ar

Aller au sommaire du numéro

Éditeur(s)

Tangence

ISSN

1189-4563 (imprimé)

1710-0305 (numérique)

Découvrir la revue

Citer cet article

Milot, L. (1992). L'envers fatal de la curiosité : lecture sémiotique d'un fait divers passionnel. Tangence, (37), 70-80. https://doi.org/10.7202/025726ar d'utilisation que vous pouvez consulter en ligne.

https://apropos.erudit.org/fr/usagers/politique-dutilisation/ 


\section{L'envers fatal de la curiosité. Lecture sémiotique d'un fait divers passionnel Louise Milot*}

\section{En guise d'introduction: corpus et méthode}

Convenir de lire sémiotiquement . Une famille massacrée à la hache * revient à affirmer le caractère résolument construit d'un tel récit, son statut fictionnel.

Le postulat de départ - qui sous-tendait également la lecture déjà ancienne d'un reportage de Paris $M a t c b^{1}$ - suggère qu'audelà des intentions, et éventuellement des gaucheries d'un reporter, le récit d'un quelconque événement, d'un fait divers, obéit à des règles en plusieurs points analogues à celles qui régissent tout discours et d'où l'on peut déduire une intentionnalité 2 .

Aborder sémiotiquement n'importe quel spécimen de ce type de texte conduit alors, indépendamment de la question du genre en tant que tel sur laquelle la sémiotique n'a pas de prise directe, à la problématique générale de l'énonciation, soit à la mise au jour d'un projet énonciatif global pour ce discours. Comment le Sujet d'énonciation - instance que la sémiotique greimassienne ${ }^{3}$ postule responsable d'un énoncé comme celui d'*Une famille massacrée à la hache. - dit-il ce qu'il dit, à travers la mise en discours d'un adolescent présumé coupable du massacre de Rochester, et selon la description que fait des événements un journaliste du Journal de Montréal? Tel est le seul cadre linguistique à l'intérieur duquel nous souhaitons et pouvons interroger le texte, étant entendu que dans ce cadre tout de même assez large, l'éventail des interrogations peut l'être aussi, ce qui oblige à préciser l'angle d'ouverture.

\section{Université Laval.}

1 Louise Milot, Sémiotique anthropomorphe us sémiotique textuelle. L'apparition du pape à Lourdes *, Protée, vol. XI, no 3, automne 1983, p. 6-18.

2 C'est-à dire une direction, un sens. Voir Louise Milot, Comment lire un texte de fiction *, dans H.J. Greif (éd.), Le risque de lire, Québec, Nuit blanche, 1988, p. 11-36

3 C'est cette sémiotique, dite de l'École de Paris, qui constitue l'arrièreplan méthodologique du présent article. 
Du point de vue du corpus d'abord, ne sera pris en considération que ce que nous considérons comme le "corps* de l'article, soit les quatre paragraphes à partir de *Le garçon* jusqu'à la fin. C'est donner à la photographie, à la rubrique qui la commente, au titre de l'article et au paragraphe introductif en caractères gras le statut de segments paratextuels en partie autonomes, s'articulant dans un rapport paradigmatique avec les paragraphes du corpus d'analyse, plutôt qu'en linéarité avec ceux-ci. Nous pourrons avoir recours à l'un ou l'autre de ces paratextes en cours d'analyse ou à son terme, mais aux seules fins d'en valider ou d'en orienter la description. Un tel découpage est discutable: nous le justifions pour des raisons de cohérence et d'économie et, dans le cas de la photographie, pour éviter le recours systématique à la sémiotique visuelle.

C'est donc la construction de ces quatre paragraphes de référence que nous tenterons de mettre en évidence, dans une lecture qui sera fidèle à deux habitudes méthodologiques dont chacune a à voir avec le principe de présupposition. Premièrement, un texte est lisible à partir de sa fin et son organisation narrative gagne à être dépliée en fonction de cette finale ${ }^{4}$; deuxièmement, pour cerner l'organisation narrative en question, l'efficacité de la séquence narrative canonique en quatre phases mise au point par A. J. Greimas 5 est retenue comme pertinente, dans la mesure où nous la confrontons à l'énonciation du texte, plutôt qu'à son énoncé, tendant à la faire servir à la prise en compte de la dimension figurative du texte, et non seulement de son organisation narrative au sens strict ${ }^{6}$.

\section{De la scène finale à l'organisation narrative}

Dans le contexte d'un développement assez récent de la sémiotique greimassienne, soit l'intégration à la théorie de la dimension passionnelle des textes ${ }^{7}$, le lecteur est frappé d'entrée

4 Louise Milot, "Comment lire un texte de fiction", loc. cit.

5 Voir l'article "schéma narratif dans A. J. Greimas et J. Courtés, Sémiotique. Dictionnaire raisonné de la théorie du langage, Paris, Hachette, 1979, p. 244-247.

6 Pour une explication détaillée de cette pratique, nous renvoyons à un article à paraître de L. Milot et F. Roy dans la revue Protée (hiver 1993), Les schémas dans le discours (D. Bertrand et $\mathrm{L}$. Milot éds).

7 Disons simplement que la parution, en 1991, de l'ouvrage d'A. J. Greimas et J. Fontanille Sémiotique des passions. Des états de cboses aux 
de jeu par l'épisode final de ce fait divers, qui postule la curiosité du père à l'endroit d'une cassette récemment achetée par son fils, curiosité qui se serait avérée pour lui fatale. La passion de la curiosité, selon Herman Parret, n'aurait pas pour finalité la vérité en tant que telle, mais la réalisation d'une vérité qui présente pour le curieux de l'intérêt, de l'importance et de l'utilité ${ }^{8}$. L'épisode final se révèle dès lors énigmatique à plus d'un titre, mais il semble en tout premier lieu dérisoire, accolant au tragique d'un quadruple crime une motivation qui pourrait être de l'ordre du quotidien le plus anodin dans les rapports d'un père et de son fils adolescent. Aussi en vient-on à se demander, pour reprendre la définition logique de Parret, ce qu'il y avait de si intéressant, de si important et de si utile pour le père à vouloir écouter cette fameuse cassette, et pour le fils inversement, à retenir l'information'. Mais ce n'est pas là évidemment la bonne question à poser à cet épisode de fiction. Ce qui est sûr en tout cas, c'est que dans la mesure où il scelle la clôture du texte, il provoque le lecteur à essayer de le motiver sémiotiquement, ce qui ne peut être fait qu'en relation avec l'ensemble textuel. En d'autres mots, si tant est qu'il s'agit là, dans cette curiosité interdite et malheureuse, de la sanction que construit la narration du fait divers, il faut s'interroger sur ce qui a mené le texte à cette curiosité, et plus exactement sur la nature de la performance qu'une telle finale vient sanctionner.

Le corpus d'analyse se présente à la lecture en quatre paragraphes d'importance comparable qui peuvent être désignés de la façon la plus neutre possible par les intitulés suivants:

1. l'interrogatoire du garçon;

2. le point de vue prospectif de la justice sur les sanctions souhaitées;

3. la scène de la découverte du crime;

4. le point de vue rétroactif des copains du garçon quant aux motivations possibles.

états d'âme (Paris, Seuil) amène les sémioticiens à interroger leur pratique actuelle, centrée sur les dimensions pragmatique et cognitive, et à y intégrer éventuellement la dimension thymique.

8 Herman Parret, Les passions. Essai sur la mise en discours de la subjectivité, Bruxelles, Pierre Mardaga, 1986, p. 172.

9 La curiosité comporte un sème d'savidité. qui n'est pas sans lien avec l'avarice. Voir A. J. Greimas et J. Fontanille, op.cit., p. 118. 
Il est tentant de corréler ces quatre segments pré-découpés par le texte lui-même aux quatre phases d'une séquence narrative classique. C'est faire l'hypothèse que ce qui est évoqué dans le premier paragraphe - en gros la référence au garçon et à son interrogatoire - tiendrait lieu de mise en place des enjeux textuels (manipulation), par rapport aux intentions arrêtées de l'appareil judiciaire au second paragraphe, qui seraient vues comme nécessaires (compétence) à la performance: celle-ci coïnciderait alors avec la référence à l'état des lieux du meurtre au troisième paragraphe; nous avons déjà proposé ci-dessus d'assimiler le paragraphe final à la sanction. On voit un peu, à travers cette hypothèse, ce que sous-tend la reconstruction d'une narrativité d'ordre énonciatif plutôt qu'énoncif, telle que nous l'avons évoquée ci-dessus. Le texte y est reçu tel quel, dans son aplat, c'est-à-dire tel qu'il est écrit et dans l'ordre de présentation de l'article, quels qu'en soient par ailleurs la gratuité ou l'aléatoire apparents. Et l'analyse en dispose, dans un premier temps, comme d'une structure. Nous essaierons d'étayer cette hypothèse narrative par la mise en relief d'un certain nombre de régularités ou d'inversions discursives d'une phase à l'autre. Les résultats seront présentés en commençant par l'examen de la phase de performance, ce qui permettra d'anticiper l'identification d'un programme énonciatif.

\section{II.1. La performance: une occultation décisive}

Partons d'un effet de sens assez général: le peu d'importance relative accordée, dans le mot à mot du récit, au personnage de l'adolescent lui-même, par rapport aux nombreuses manifestations actorielles des représentants de la justice dont sont déclinés et les noms (MM. Raymond Schmitz et Charles Von Wald) et les titres (procureur du comté d'Olmsted et shérif du même comté), et des autres membres de la famille, tous identifiés par leur prénom et par leur âge. L'adolescent, lui, le moteur de toute cette histoire pourtant, n'est jamais nommé ${ }^{10}$, mais désigné tour à tour par les vocables :le garçon* (deux fois), "l'adolescent meurtrier * (une fois), * il " (six fois) ou *lui * (une fois).

10 Nous ne prenons évidemment pas en considération, dans une telle analyse, la contrainte de la confidentialité, qui pourrait jouer dans le cas d'un mineur. D'autant qu'avec la photographie et l'identification complète de la famille, il n'y a plus aucune confidentialité qui tienne. 
Notre proposition, précisément, est que la performance du texte consisterait à distinguer, puis à isoler l'adolescent meurtrier du reste de sa famille, voire à disposer de cet acteur, à l'éliminer de l'énonciation textuelle en somme, tout comme le personnage a lui-même disposé de sa famille dans l'énoncé. Cette avancée requiert quelque explication.

Faisons d'abord remarquer que le troisième paragraphe se construit à partir de deux * découvertes * parallèles (le même lexème est d'ailleurs convoqué dans les deux cas) qu'il incombe à la lecture de mettre en relation: la découverte des quatre cadavres, et la découverte d'une hache ensanglantée. Un certain nombre d'oppositions comparables surdéterminent ce fond commun: leur caractéristique générale serait de marquer la mise en discours (l'énonciation) de la première découverte d'une relative euphorie textuelle, quoiqu'il soit pourtant question de cadavres, par rapport à la seconde, marquée de dysphorie. D'une part, en effet, les cadavres sont favorisés par la loi du nombre (ils sont quatre), se démarquant de l'occurrence unique de la hache. Ils sont en outre, et paradoxalement, parfaitement conformes aux normes sociales: identifiés par leur nom et par leur âge, comme nous l'avons déjà fait remarquer, convenablement vêtus ( «ils portaient encore leurs vêtements de nuit •) et comme à l'abri d'un quelconque désordre, par opposition à l'unique qualité divulguée pour la hache, soit d'être ensanglantée, qualité inconvenante et négative, et, partant, marginalisante. Un écart du même ordre recouvre les localisations spatiales respectives des deux ensembles découverts: les cadavres, dirait-on, légalement et ouvertement disposés "dans leur maison de Cascade Township, dans la banlicue de Rochester * - leur situation bénéficiant de la même abondance descriptive que les cadavres eux-mêmes -, la hache au contraire, "dans la cave de la maison", comme enfouie.

Deux ensembles parallèles sont ici résolument distingués et isolés l'un de l'autre. L'un de ces ensembles renvoie évidemment à la collectivité familiale sur laquelle les traces d'agression, curieusement, sont inexistantes; l'autre pourrait concerner l'adolescent, si l'on convient que la figure de la hache tient lieu, métonymiquement, du personnage qui en avait fait son arme. Entre les cadavres et l'arme du crime toutefois, on ne peut qu'enregistrer (et peut-être trouver curieux) que l'écriture du paragraphe central choisisse de ne pas tenir compte, dans la description du paysage du crime, du protagoniste essentiel qu'est l'adolescent lui-même. L'absence de 
cet acteur de la seule véritable s scène - du texte paraît d'autant plus significative que sur le strict plan de la vraisemblance, rien ne permet de croire - le premier paragraphe suggère peut-être même le contraire - que le garçon avait déserté les lieux de son crime. Quoi qu'il en soit, en laissant la place du garçon vacante, en creux, dans l'ombre, entre les effets de son acte et sa représentation métonymique, le texte du troisième paragraphe, c'est notre hypothèse, signifie bien qu'il s'agit moins d'enregistrer une absence que de nier une présence. Aussi est-on justifié de revenir en arrière pour tenter de retracer les préalables de cette occultation.

\section{II.2. La manipulation : mise en scène de la curiosité}

Le personnage du garçon avait pourtant fait une entrée fulgurante au premier paragraphe dont il était, littéralement et sans contredit, le centre: "en cours d'interrogatoire à la poste centrale de Rochester " (nous soulignons). Il faut bien voir que la mise en discours de sa présence, à ce premier stade - seul moment du texte où il est décrit directement par le narrateur, sans intermédiaire ${ }^{11}$ - , apparaît rétroactivement prendre par anticipation l'exact contrepied des modalités de son élimination au paragraphe 3. $\mathrm{Si}$, à ce dernier endroit, le meurtrier n'était manifestê que métonymiquement (par l'arme du crime), au premier paragraphe, sa présence est au contraire on ne peut plus insistante et à la limite de l'excès. Le garçon métaphorise le crime, dont il mime dans son travestissement et la méthode, et le résultat. Figurée par une tête aux cheveux en partie rasés, en partie teints, comme si le personnage avait été lui aussi séparé en deux par la hache dont il s'est servi contre sa famille, l'allure du garçon subsume et synthétise à la fois (et aussi bien) les cadavres, désormais "rasés" de leur vie, et la hache "teinte - de sang. La force d'évocation de cet amalgame, sorte de débordement sémantique impliquant à la fois l'arme du crime, les cadavres et le criminel lui-même, dans l'actorialisation spectaculaire du garçon seul susceptible de faire tenir un tel univers en syncrétisme, est certes le volet le plus fort du contrat manipulatoire de ce texte, et celui précisément dont on peut faire l'hypothèse que le récit du fait divers, en le posant d'entrée de jeu, manifeste en même temps qu'il s'agit là d'un

11 Au paragraphe final, nous le verrons, il réapparaîtra, mais à travers l'écran métadiégétique de la narration d'un autre. 
contenu à inverser. Dans la seconde moitié de la seule longue phrase qui le constitue, le premier paragraphe s'applique d'ailleurs déjà à déloger le garçon du temps et de l'espace mythiques du crime où il s'était modelé à l'image de sa famille "avant que les cadavres ne soient découverts ", vers un temps et un espace plus terre-à-terre qui ne seront plus les siens mais ceux de la justice, où le garçon n'est plus qu'un individu parmi d'autres, dans *une ville de 60000 habitants .

Le premier paragraphe présente ainsi véritablement les enjeux d'une manipulation (au sens sémiotique du terme et sans connotation péjorative), c'est-à-dire la tentative d'imposer un nouveau contrat énonciatif. En réponse à la question embarrassante que pose à ses interlocuteurs l'allure même du garçon, véritable objet de *curiosité *, le texte s'applique visiblement à déplacer les enjeux vers ce que nous appellerions une neutralisation de l'énigme, figurée ici par l'interrogatoire banal et classique du présumé coupable.

\section{II.3. La compétence : le savoir judiciaire}

L'article en effet ne révélera rien des réponses ou des silences du garçon. En lieu et place, le second paragraphe, dont le ton est particulièrement décidé et affirmatif, fournit bien une réponse, mais c'est celle de l'appareil judiciaire, lequel décline ses intentions. La déclaration officielle (elle est citée textuellement) du procureur (celui-ci dûment nommé et identifié), la clarté de ses objectifs (. introduire une demande [...] et nous demanderons ensuite [...].) construisent un cadre cognitif non équivoque, l'assertion d'un savoir en quoi nous voyons une compétence. Le contenu de ce discours, qui aboutit à ce que le garçon *soit jugé comme un adulte, amorce déjà, de notre point de vue, le processus de sortie de sa famille pour le garçon: s'il est décrété adulte, il perd forcément son statut d'enfant ${ }^{12}$. Nous avons déjà suggéré que ce soit ce processus qui soit donné comme concrétisé au paragraphe 3, à travers l'absence du protagoniste de la scène d'un meurtre dont il est par ailleurs le responsable.

12 Sur ce point précis, on peut attirer l'attention sur un choix lexical du commentaire sous la photographie, qui nous semble recouvrir de qui vient d'être dit: :Quatre des membres de cette famille y est opposé à -celui , pour désigner le garçon. 
Nous revenons à peu près à notre point de départ, soit le sens de la finale, et sommes mieux en mesure, espérons-le, de répondre à la question alors soulevée concernant le dernier paragraphe: comment peut-on dire qu'il sanctionne très exactement le déroulement textuel qui précède?

\section{II.4. La sanction: le bien-fondé de l'élimination}

C'est comme si le paragraphe final repassait sur toutes les traces importantes des paragraphes précédents - la mise en discours du garçon, de l'appareil judiciaire, de la famille --, pour en répéter ou en confirmer la pertinence. Pas étonnant au fond, dans la mesure où la sanction est précisément une opération de véridiction portant sur les choix textuels antérieurs.

Absent de l'article depuis le premier paragraphe, le personnage du garçon semble pouvoir être ramené sans danger au paragraphe final: c'est qu'en effet il n'est plus le même, on dira en sémiotique qu'il a été recatégorisé. Remarquons tout d'abord qu'il est de nouveau représenté dans son double rôle d'enfant -ses amis étant désignés comme des “écoliers * - et de meurtrier, *adolescent meurtrier , après avoir été pourtant dépouillé de ces deux mêmes fonctions, respectivement aux paragraphes 2 et 3 . Mais notons du même coup le recul de sa position par rapport au point de départ. Ce n'est plus lui désormais qui est interrogé par l'autorité - ici le shérif du comté, M. Charles Von Wald - mais ce sont ses copains, et plus précisément :[1]'un d'entre eux", devenu en quelque sorte son substitut; et c'est de ce double, de ce simulacre, dont le lecteur devra se satisfaire pour connaitre les motivations éventuelles du garçon. Ce qui est dit se ramène par ailleurs à deux choses: • il avait des problèmes avec ses parents., nommément *avec son père en raison d'une cassette qu'il avait achetée et qu'il ne voulait pas que son père écoute .

Sans revenir sur le dérisoire, déjà évoqué, d'un tel motif, dans le texte s'entend - car les motifs réels de l'adolescent n'ont pas pour nous de pertinence - , regardons-y de plus près. Voudrat-on nous concéder que si l'enfant réapparaît, et face à son père/ ses parents, c'est dans un type de relations familiales que nous qualifierions de verticales, et à l'inverse des stéréotypes culturels habituels. Dans notre culture en effet, et jusqu'à nouvel ordre, il revient aux parents d'avoir ou de ne pas avoir des problèmes avec leurs enfants - et non l'inverse -; et de même, du père ou 
du fils, c'est plus normalement le premier qui détient le pouvoir de surdétermination consécutif tant au pouvoir d'achat, qu'au pouvoir d'interdiction et de discrétion. Le fait, à la fin du texte, de montrer le fils dans cet état un peu excessif d'une absolue initiative concernant le jugement porté sur ses parents - *il avait des problèmes avec ses parents * - le pouvoir d'achat - «il avait acheté *-, d'interdiction - *il ne voulait pas * - et de discrétion - "que son père écoute * - confirme bien la légitimité de tout ce qui précède, essentiellement ce fait qu'on ait dû retirer au jeune meurtrier son droit d'appartenance à sa famille.

Aussi dirons-nous que si le texte reconvoque avec autant de complaisance, à la fin, "l'adolescent meurtrier "13, c'est qu'à cette étape justement il n'y a plus aucun risque à le faire. Le dernier paragraphe ne fait qu'établir - c'est le sens même de la sanction - le bien-fondé du trajet qui précède. Quelque paradoxal que cela puisse paraître en effet, l'intérêt de la finale ne réside aucunement dans le fait qu'elle fournisse aux lecteurs du Journal de Montréal les motivations plausibles, encore moins réelles, du geste étonnant du garçon. Il s'agit plutôt d'établir la véridiction de l'énoncé fictif que construit forcément tout discours. Or ici, ce que le texte conclut, nous semble-t-il, est que le comportement de l'adolescent lui-même, qui avait le premier miné, dès avant le meurtre, le sens habituel des relations parents/enfants, confirme tout à fait l'autoritê judiciaire à le traiter en adulte, c'est-à-dire à lui dénier le statut de membre d'une famille, et dans son cas le statut de mineur.

\section{Bilan de l'analyse}

Il pourrait être opportun de rappeler à la rescousse de cette rapide analyse, avant de terminer, les quelques ensembles paratextuels écartés jusqu'ici pour des raisons de méthode: le paragraphe d'introduction en gras, le titre et la photo. Ne serait-ce que pour indiquer la possibilité de leur homologation avec ce que nous avons proposé comme contenu principal du fait divers.

Le paragraphe d'introduction, nous n'y insisterons pas, semble corroborer assez clairement l'analyse proposée ici, même si nous reconnaissons bien évidemment que ces quelques lignes

On n'en a jamais autant appris à son sujet. 
appelleraient elles aussi un examen plus détaillé. Tenons-nous-en au fait qu'en s'ouvrant sur le lexème .Un adolescent * et en s'achevant à la fois sur l'assertion d'un jugement en tant qu'adulte et sur la dénégation du statut de mineur, ce préliminaire affiche la même direction que l'article lui-même, déportant l'enfant de son identité de membre jeune d'une famille, la sienne, à celle de membre adulte d'une collectivité anonyme.

Quant au titre «Une famille massacrée à la hache *, sous des dehors banals qui pouvaient ne pas retenir l'attention au point de départ de l'analyse, on conviendra qu'au terme de celle-ci, il devient gros d'évidence. Si "une famille " a été massacrée, c'est que le meurtrier n'en faisait pas partie, de cette famille, sans quoi il aurait été massacré lui aussi. On voit bien que le titre n'est textuellement correct, c'est-à-dire cohérent, que si a été préalablement mise en marge une quelconque appartenance familiale du meurtrier, ce que nous croyons avoir démontré.

Mais c'est la photographie qui constitue sans doute l'élément le plus surprenant et le plus troublant de l'ensemble paratextuel. La famille y est représentée littéralement dominée, comme par anticipation, par "celui qu'on aperçoit en haut à droite" dit le commentaire, et qui en effet occupe le sommet de la pyramide. Sa tenue vestimentaire l'isole déjà ${ }^{14}$. Se démarquant tout autant du couple sour-père, dont les encolures en $\times V$ * se répondent, que du couple frère-mère, dont les vêtements vraisemblablement très clairs blanchissent le coin gauche de la photo, le polo rayé du garçon est déjà une marginalisation du fait de ne se retrouver chez aucun partenaire. Mais il rejoint en outre, dans l'alternance de ses hachures, tant la hache elle-même évidemment, que le personnage divisé dans lequel le jeune meurtrier s'était lui-même transformé d'entrée de jeu, et qui constitue somme toute la seule représentation personnelle du personnage par lui-même dans le texte. Ne peut-on même aller jusqu'à dire que le fait de l'opposition du garçon, focalisée principalement sur le père, est répercutée sur la photo dans l'opposition très marquée de la ligne verticale unique formée par la cravate foncée du père, et des lignes horizontales nombreuses du chandail du garçon. Comme si tant la performance de l'énoncé - dominer sa famille jusqu'à la destruction - que celle de l'énonciation - être exclus de cette

14 Je remercie Catherine Lamy, étudiante au doctorat, qui a attiré mon attention sur les jeux vestimentaires de cette photographie. 
80

famille - se trouvaient proclamées par la situation et l'allure du meurtrier sur la photo de famille.

\section{Pour conclure}

En attirant l'attention, au début de cet exercice, sur la passion de la curiosité, nous comptions revenir sur l'image initiale de l'adolescent transformé en objet de curiosité, pour tenter de relire le texte à travers elle tout en y reliant la curiosité finale du père. Il aurait semblé intéressant de rebaliser le trajet du texte - et, partant, de questionner la pertinence de l'analyse - au moyen de cette passion, si tant est que la dimension thymique des textes risque de se révéler fondamentale. Mais il aurait certes fallu rétablir, préalablement, les maillons intermédiaires, qu'on pourrait désigner temporairement comme l'absence totale de curiosité à l'endroit de l'adolescent, au paragraphe 3, et au contraire l'avidité compulsive ${ }^{15}$ de la demande qu'on en fasse un adulte au paragraphe 2. Il s'agira là d'un autre travail, que d'aucuns pourront trouver aussi étonnant que le présent fait divers, pour ne pas dire aussi * curieux $* 16$.

15 Voir ci-dessus la note 9.

16 Ce travail à la lumière de la passion de la curiosité a fait, depuis, l'objet d'une communication au colloque * Rêves bibliques / Passions littéraires ", Congrès de l'ACFAS, mai 1992, Université de Montréal. 